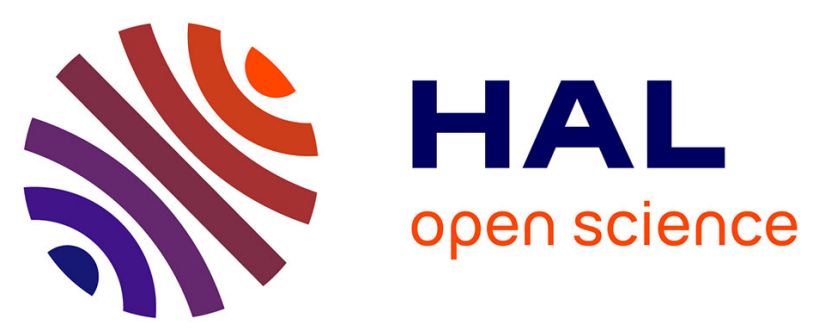

\title{
An EMG-Driven Biomechanical Model That Accounts for the Decrease in Moment Generation Capacity During a Dynamic Fatigued Condition
}

Guillaume Rao, Eric Berton, David Amarantini, Laurent Vigouroux, Thomas

S. Buchanan

\section{To cite this version:}

Guillaume Rao, Eric Berton, David Amarantini, Laurent Vigouroux, Thomas S. Buchanan. An EMG-Driven Biomechanical Model That Accounts for the Decrease in Moment Generation Capacity During a Dynamic Fatigued Condition. Journal of Biomechanical Engineering, 2010, 132 (7), 10.1115/1.4001383 . hal-01442063

\section{HAL Id: hal-01442063 https://hal.science/hal-01442063}

Submitted on 15 Nov 2017

HAL is a multi-disciplinary open access archive for the deposit and dissemination of scientific research documents, whether they are published or not. The documents may come from teaching and research institutions in France or abroad, or from public or private research centers.
L'archive ouverte pluridisciplinaire HAL, est destinée au dépôt et à la diffusion de documents scientifiques de niveau recherche, publiés ou non, émanant des établissements d'enseignement et de recherche français ou étrangers, des laboratoires publics ou privés. 
Guillaume Rao ${ }^{1}$

Institute of Movement Sciences, University of the Mediterranean,

UMR CNRS 6233,

163, Avenue de Luminy,

13288 Marseille Cedex 09, France;

Department of Mechanical Engineering,

University of Delaware,

126 Spencer Laboratories, Newark, DE 19716

e-mail: guillaume.rao@univmed.fr

Eric Berton

Institute of Movement Sciences,

University of the Mediterranean,

UMR CNRS 6233,

163, Avenue de Luminy,

13288 Marseille Cedex 09, France

e-mail: eric.berton@univmed.fr

David Amarantini

Universite de Toulouse,

UPS, LAPMA,

118, Route de Narbonne,

F-31062 Toulouse Cedex 09, France

e-mail: david.amarantini@cict.fr

\section{Laurent Vigouroux}

Institute of Movement Sciences,

University of the Mediterranean, UMR CNRS 6233

163, Avenue de Luminy,

13288 Marseille Cedex 09, France e-mail: laurent.vigouroux@univmed.fr

\section{Thomas S. Buchanan}

Fellow ASME

Department of Mechanical Engineering, University of Delaware,

126 Spencer Laboratories, Newark, DE 19716 e-mail: buchanan@udel.edu

\section{An EMG-Driven Biomechanical} Model That Accounts for the Decrease in Moment Generation Capacity During a Dynamic Fatigued Condition

Although it is well known that fatigue can greatly reduce muscle forces, it is not generally included in biomechanical models. The aim of the present study was to develop an electromyographic-driven (EMG-driven) biomechanical model to estimate the contributions of flexor and extensor muscle groups to the net joint moment during a nonisokinetic functional movement (squat exercise) performed in nonfatigued and in fatigued conditions. A methodology that aims at balancing the decreased muscle moment production capacity following fatigue was developed. During an isometric fatigue session, a linear regression was created linking the decrease in force production capacity of the muscle (normalized force/EMG ratio) to the EMG mean frequency. Using the decrease in mean frequency estimated through wavelet transforms between dynamic squats performed before and after the fatigue session as input to the previous linear regression, a coefficient accounting for the presence of fatigue in the quadriceps group was computed. This coefficient was used to constrain the moment production capacity of the fatigued muscle group within an EMG-driven optimization model dedicated to estimate the contributions of the knee flexor and extensor muscle groups to the net joint moment. During squats, our results showed significant increases in the EMG amplitudes with fatigue $(+23.27 \%$ in average) while the outputs of the EMG-driven model were similar. The modifications of the EMG amplitudes following fatigue were successfully taken into account while estimating the contributions of the flexor and extensor muscle groups to the net joint moment. These results demonstrated that the new procedure was able to estimate the decrease in moment production capacity of the fatigued muscle group. [DOI: 10.1115/1.4001383]

Keywords: EMG, biomechanical model, muscle fatigue, muscle force, frequency shift

\section{Introduction}

Muscular fatigue is an inevitable feature of muscle activation, such as that which occurs during sporting competitions, rehabilitation exercises, or repetitive tasks (e.g., lifting and material handling). The fatigue starts at the beginning of the muscle activation and is commonly defined as a loss of force production capacity associated with an increase in the perceived level of effort [1]. The multiple physiological mechanisms underlying the decrease in force production capacity have been studied extensively and encompass central fatigue with diminished muscle activation [2] or peripheral fatigue with an increased concentration of inorganic phosphate or a failure in excitation-contraction coupling [3,4]. Changes in the electromyographic (EMG) signal have also been reported in the presence of fatigue. To compensate for the loss of force production capacity, previous work reported an increase in the EMG amplitude during submaximal isometric activities. Consequently, force decreases for constant levels of EMG during

\footnotetext{
${ }^{1}$ Corresponding author.

Contributed by the Bioengineering Division of ASME for publication in the JouRNAL OF Biomechanical EngineERING. Manuscript received February 11, 2008; final manuscript received April 14, 2009; accepted manuscript posted March 8, 2010; published May 14, 2010. Assoc. Editor: Avinash Patwardhan.
}

maximal voluntary activations [1]. Related to the development of muscle fatigue, the EMG frequency content is also modified as the mean and median frequencies have been shown to decrease $[1,5,6]$. During dynamic (i.e., force-varying and/or nonisometric) contractions, the EMG signal has been described as nonstationary because of changes in its frequency content through time [5,6]. Due to these nonstationarities, time-frequency methods that do not rely on a local quasi-stationarity assumption (e.g., Choi-Williams distributions or wavelet transforms) are better suited to the spectral analysis of the EMG signal during fatiguing and/or dynamic contractions than the fast Fourier transforms $[5,6]$.

The muscular redundancy of the human body prevents one from finding a unique combination of muscle forces leading to the net joint moment [7]. Most advanced biomechanical models use geometrical and physiological data (force-length and forcevelocity relationships, moment arms, physiological cross sectional area (PCSA), and muscle length), kinematics, dynamics, EMG, and mechanical laws of motion as inputs to optimization processes in order to resolve the redundancy issue [8-10]. These models use the EMG amplitudes as direct or indirect information to compute muscle forces or muscle group contributions to the net joint moment. Despite the quasi-permanent presence of muscle fatigue during daily activities, few studies have attempted to take into account its presence when investigating muscle forces or their 
contributions to the net joint moment. Indeed, the major limitation of using the EMG data of fatigued muscles in EMG-driven biomechanical models is the altered EMG-force relationship. A single study reported a method to take into account the changes in the EMG amplitudes due to fatigue during an isokinetic exercise [11]. This method was used as a part of an EMG-driven biomechanical model to estimate the modifications of spinal loading due to fatigue. However, this method required the movement to be performed on a dynamometer because a direct measurement of the force produced during the motion was needed. Its utility would be greatly improved if such a method could be applied to functional nonisokinetic movements.

The aim of the present study was to develop an EMG-driven biomechanical model to obtain the contributions of flexor and extensor muscle groups to the net joint moment during a nonisokinetic functional movement (squat exercise) performed in nonfatigued and in fatigued conditions. A methodology that aims at balancing the decreased muscle moment production capacity following fatigue was developed. This procedure aims at, first, assessing the changes in the activation level due to the presence of muscle fatigue (i.e., the changes in the EMG-moment relationship) and, second, taking these changes into account while estimating the contributions of the agonist and antagonist muscle groups to the net joint moment. We expected higher EMG amplitudes for the fatigued than for the nonfatigued condition for the same motion. When applying the original methodology developed in this study and assuming few changes in muscle activation patterns, we hypothesized identical outputs of the EMG-driven model (i.e., the contributions of the flexor and extensor muscle groups to the net joint moment) between both conditions. Indeed, given identical inputs in terms of kinematics and dynamics data, increased EMG amplitudes of the agonist muscles under fatigue (required to perform the task due to the low moment production capacity) would be balanced by the methodology presented here.

\section{Methods}

2.1 Subjects. Nine male subjects participated in this study. Mean ( \pm s.d.) age, height, and mass were, respectively, 26.9 ( \pm 3.2 years $), 1.75( \pm 0.05 \mathrm{~m})$, and $70.6( \pm 6.2 \mathrm{~kg})$. All subjects were free of known injuries. The project was approved by the University Review Board and all subjects gave written consent after being informed of the experimental procedures.

2.2 Experimental Protocol. The experimental design was comprised of four consecutive steps: an isometric maximal voluntary contraction, 11 cycles of dynamic half squats in a nonfatigued condition, an isometric fatigue session, and 11 cycles of dynamic half squats in a fatigued condition.

The first step involved isometric maximal voluntary contractions. The subjects were standing on a force plate and anchored to the ground using four inextensible ropes. Rope lengths were adjusted so that knee angles were close to $90 \mathrm{deg}$ (Fig. 1). The subjects were instructed to produce the greatest vertical force during $4 \mathrm{~s}$ while maintaining their trunks as vertical as possible. In order to obtain a maximal performance, a visual feedback of the performance was provided through an oscilloscope and subjects were given verbal encouragement. The maximal value within three trials of maximal voluntary force (MVF) measured by the force plate was used in the third step of the experiment.

The second step examined dynamic half squats in the nonfatigued condition. The subjects performed 11 cycles of dynamic half squats (beginning in an upright posture and bending the knees until the thighs were parallel to the ground, then going back up) loaded with $20 \%$ of their bodyweight. The bar was positioned on the subjects' shoulders. A piece of wood was located below the heels and no motion of the heels was allowed. A metronome was used to keep constant the duration of each squat cycle $(0.5 \mathrm{~Hz}$ frequency). Online verbal feedbacks were given to the subjects in

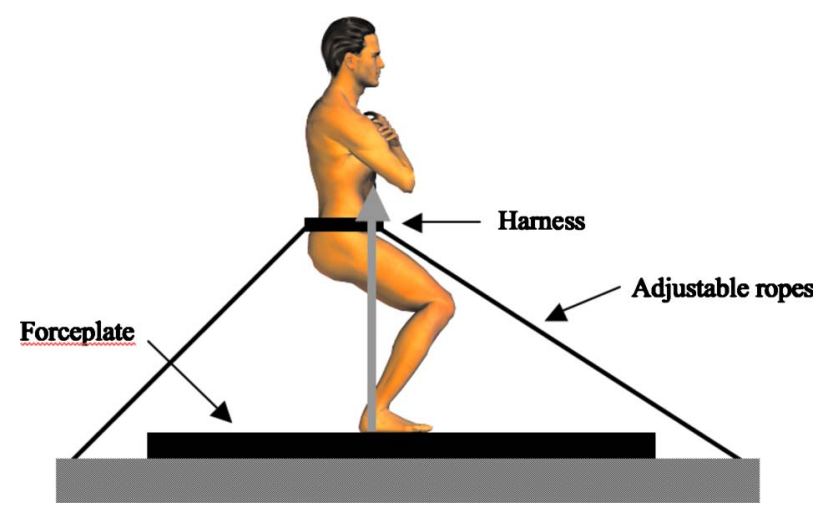

Fig. 1 Typical posture of a subject during the MVC and the isometric fatigue tasks

order for them to control their motions and perform the same squat movements in nonfatigued and fatigued conditions.

The third step was an isometric fatigue session. The subjects had to sustain $60 \%$ MVF for as long as possible to create fatigue in the knee extensor muscle group. This isometric fatigue protocol was performed in the same posture as the MVF trials. A visual feedback of the force to sustain was provided using an oscilloscope. The fatigue session was terminated as soon as the force level dropped below 40\% MVF.

Finally, the fourth step consisted of dynamic half squats in the fatigued condition. Immediately after the end of the fatigue session, the subjects were loaded with $20 \%$ bodyweight and performed another block of 11 squat cycles. The squat cycle duration was also controlled $(0.5 \mathrm{~Hz}$ pace).

2.3 Data Acquisition. Kinematic data were recorded at 120 $\mathrm{Hz}$ using a six camera Vicon 624 system. Trajectories of the foot, shank, thigh, trunk, and barbell segments were recorded from ten passive markers placed over the following landmarks on the right side of the body: the fifth metatarsal head, the lateral malleolus, the lateral femoral condyle, the great trochanter, the head of the clavicle, the epicondyle of the elbow, the wrist, the jaw, the vertex of the head, and the middle of the barbell. The ground reaction data were sampled at $1560 \mathrm{~Hz}$ from a six component AMTI force platform. As recommended in Refs. [8,12], electromyographic data for the gastrocnemius medialis (ga), biceps femoris (bf), rectus femoris (rf), and vastus medialis (vm) muscles were recorded at $1000 \mathrm{~Hz}$ using a Biopac MP 150 system (gain=500, input impedance $=1.0 \mathrm{M} \Omega$, common mode rejection ratio $(\mathrm{CMRR})$ $=110 \mathrm{~dB}$, noise $=0.5 \mu \mathrm{V}$, resolution $=16$ bits $) . \mathrm{Ag} / \mathrm{Ag}-\mathrm{Cl}$ bipolar surface electrodes were placed over bellies of the selected muscles with a $2 \mathrm{~cm}$ center-to-center interelectrode distance. Skin preparation and electrode placements were done following the recommendations of the SENIAM group [13]. A TTL signal generated by the EMG acquisition device was later used to synchronize the EMG and the kinematics and dynamics data.

2.4 Data Processing. Ground reaction data were filtered using a fourth-order zero phase-lag Butterworth filter with a $10 \mathrm{~Hz}$ net cutoff frequency. Marker trajectories were smoothed using a constrained cubic smoothing spline procedure (MATLAB, SPLINE TOOLBOX, version 3.2.2). Angular time series of the ankle, knee, hip, and trunk angles were computed from smoothed Cartesian coordinates of the markers. Using cubic spline interpolation, angular kinematics, and ground reaction were resampled to $1000 \mathrm{~Hz}$ to match the EMG acquisition frequency. Values of joint angular velocities and accelerations were obtained by analytically differentiating the spline functions.

Based on the vertical velocity of the barbell, each variable of the squat cycle was normalized in time from $0 \%$ to $100 \%$. The two first and two last cycles were removed from the analysis to 
avoid electromyographic and kinematic changes due to movement initiation and termination. The seven remaining cycles were averaged to obtain one representative cycle for each variable (EMG, angular kinematics, and ground reaction) for each subject. In the nonfatigued and fatigued conditions, the knee net joint moment was computed via inverse dynamics [14] using the representative cycles of kinematics and ground reaction data as well as body segment parameters from Ref. [15]. Inverse dynamics were computed using the Langrangian formalism with the human body modeled as a planar four-link system with frictionless hinges.

2.5 Modeling. Based on the modifications of the EMG signal during the isometric fatigue session, a methodology was developed to assess the loss of moment production capacity of the fatigued muscle group during the dynamic session. This methodology was further used as a part of an EMG-driven biomechanical model to estimate the contributions of the flexor and extensor muscle groups to the knee net joint moment under muscle fatigue condition.

First, the isometric fatigue session served as a basis to create a linear relationship representing the loss of force production capacity of the fatigued muscles as a function of the decrease in the EMG mean frequency. Second, an EMG-driven model was used to estimate the flexor and extensor contributions to the knee net joint moment in the nonfatigued condition. Third, the decrease in force production capacity in the fatigued dynamic condition (further referred as the $\mathrm{K}$ coefficient) was estimated using the decrease in the EMG mean frequency between the nonfatigued and the fatigued conditions as input to the linear relationship. Finally, the $\mathrm{K}$ coefficient was used in the EMG-driven model to constrain the moment production capacity of the fatigued muscle group. These different steps will be detailed in the following paragraphs.

2.5.1 Isometric Fatigue Session. During the isometric fatigue session, the subjects had to sustain $60 \%$ of their MVF for as long as possible. During this session, angular kinematics and EMG data were analyzed within windows that were 512 points long and centered every $5 \%$ force decrease. These windows corresponded to $60 \%$ (nonfatigued condition), $55 \%, 50 \%, 45 \%$, and $40 \%$ of the MVF. Within each window, the knee angles and the root mean square (RMS) of the EMG signal were computed. Wavelet transforms with Morlet wavelets of 126 points length at the coarsest scale were used to obtain the values of the mean frequency of the myoelectric signal within each window $[16,17]$.

Force plate vertical force, RMS EMG, and mean EMG frequency values were then normalized relative to values of the nonfatigued condition. Because adaptations to fatigue may arise either on EMG or on force signals, a ratio between the normalized force plate vertical force and the normalized RMS EMG signal was computed for each analysis window (normalized force/EMG ratio). Finally, linear regression was used to fit the normalized force/ EMG ratio to the normalized mean frequency decrease. Thus, the output of the isometric fatigue session consisted of a linear relationship that represents the normalized force/EMG ratio (the force production capacity of the muscle group) as a function of the normalized mean frequency decrease.

Considering, first, that the fatigue protocol would only affect the extensor muscle group and, second, that the low activation levels of the flexor muscles would produce highly variable frequency content estimates, the linear regression was only computed for the knee extensor muscles (i.e., rectus femoris and vastus medialis muscles).

2.5.2 Dynamic Trials-Nonfatigued Case. The contributions of the knee flexor and extensor muscle groups to the net joint moment were obtained from an EMG-to-moment optimization procedure $[8,18]$. For the nonfatigued condition, the optimization problem was formulated as follows:
Find

$$
\begin{aligned}
\alpha_{\mathrm{i}}^{\mathrm{nf}}=\left\{\alpha_{\mathrm{ga}}^{\mathrm{nf}}, \alpha_{\mathrm{bf}}^{\mathrm{nf}}, \alpha_{\mathrm{rf}}^{\mathrm{nf}}, \alpha_{\mathrm{vm}}^{\mathrm{nf}}\right\}, \quad \beta_{\mathrm{j}}^{\mathrm{nf}}=\left\{\beta_{\mathrm{A}}^{\mathrm{nf}}, \beta_{\mathrm{K}}^{\mathrm{nf}}, \beta_{\mathrm{H}}^{\mathrm{nf}}\right\}, \quad \delta_{\mathrm{j}}^{\mathrm{nf}} \\
=\left\{\delta_{\mathrm{A}}^{\mathrm{nf}}, \delta_{\mathrm{K}}^{\mathrm{nf}}, \delta_{\mathrm{H}}^{\mathrm{nf}}\right\}, \quad \text { and } \quad \mathrm{w}_{\mathrm{i}}^{\mathrm{nf}}(\mathrm{t})=\left\{\mathrm{w}_{\mathrm{ga}}^{\mathrm{nf}}(\mathrm{t}), \mathrm{w}_{\mathrm{bf}}^{\mathrm{nf}}(\mathrm{t}), \mathrm{w}_{\mathrm{rf}}^{\mathrm{nf}}(\mathrm{t}), \mathrm{w}_{\mathrm{vm}}^{\mathrm{nf}}(\mathrm{t})\right\} \\
\text { that minimize: } C=\frac{1}{2}\left(\mathrm{M}_{\mathrm{K}}^{\mathrm{nf}}(\mathrm{t})-\hat{\mathrm{M}}_{\mathrm{K}}^{\mathrm{nf}}(\mathrm{t})\right)^{2}
\end{aligned}
$$

with

$$
\begin{aligned}
\hat{\mathrm{M}}_{\mathrm{K}}^{\mathrm{nf}}(\mathrm{t})= & \sum_{\mathrm{i}}\left(\alpha_{\mathrm{i}}^{\mathrm{nf}} \cdot \mathrm{w}_{\mathrm{i}}^{\mathrm{nf}}(\mathrm{t}) \cdot \mathrm{S}_{\mathrm{i}}^{\mathrm{nf}}(\mathrm{t})\right)^{T} \cdot\left[\mathbf{1}+\mathbf{E} \cdot\left(\beta_{\mathrm{j}}^{\mathrm{nf}} \cdot \Delta \theta_{\mathrm{j}}(\mathrm{t})\right)\right. \\
& \left.-\mathbf{E} \cdot\left(\delta_{\mathrm{j}}^{\mathrm{nf}} \cdot \dot{\theta}_{\mathrm{j}}(\mathrm{t})\right)\right], \quad \mathrm{i}=\{\mathrm{ga}, \mathrm{bf}, \mathrm{rf}, \mathrm{vm}\}, \quad \mathrm{j}=\{\mathrm{A}, \mathrm{K}, \mathrm{H}\}
\end{aligned}
$$

subject to

$$
\left\{\begin{array}{c}
\alpha_{\mathrm{ga}}^{\mathrm{nf}}, \alpha_{\mathrm{bf}}^{\mathrm{nf}}<0 ; \quad \alpha_{\mathrm{rf}}^{\mathrm{nf}}, \alpha_{\mathrm{vm}}^{\mathrm{nf}}>0 \\
\beta_{\mathrm{j}}^{\mathrm{nf}} \text { and } \delta_{\mathrm{j}}^{\mathrm{nf}}>0 \\
0<\mathrm{w}_{\mathrm{i}}^{\mathrm{nf}}(\mathrm{t})<1 \quad \text { as inequality constraints } \\
\hat{\mathrm{M}}_{\mathrm{ga}}^{\mathrm{nf}}, \hat{\mathrm{M}}_{\mathrm{bf}}^{\mathrm{nf}}<0 ; \quad \hat{\mathrm{M}}_{\mathrm{rf}}^{\mathrm{nf}}, \hat{\mathrm{M}}_{\mathrm{vm}}^{\mathrm{nf}}>0
\end{array}\right.
$$

The "nf" and "f" superscripts correspond, respectively, to the nonfatigued and the fatigued conditions while the indices " $\mathrm{i}$ " stand for the muscle investigated (ga, bf, rf, and vm) and the indices " $\mathrm{j}$ " represent the joint of concern (ankle, knee, and hip). Bold and italic terms stand for matrix and scalars, respectively. In Eq. (1), $\mathrm{M}_{\mathrm{K}}^{\mathrm{nf}}(\mathrm{t})$ corresponds to the knee net joint moment computed through inverse dynamics while $\hat{\mathrm{M}}_{\mathrm{K}}^{\mathrm{nf}}(\mathrm{t})$ stands for the net moment estimated using the EMG data. In Eq. (2), $\alpha_{\mathrm{i}}^{\mathrm{nf}}$ represents the isometric EMG-moment coefficient in the nonfatigued condition and $\mathrm{w}_{\mathrm{i}}^{\mathrm{nf}}(\mathrm{t})$ stands for the individual muscle gains at each time t. $S_{\mathrm{i}}^{\text {nf }}(\mathrm{t})$ contains the rectified and filtered (low-pass, fourth-order, $2.5 \mathrm{~Hz}$ net cutoff frequency) EMG data of the four selected muscles. $\hat{\mathrm{M}}_{\mathrm{K}}^{\mathrm{nf}}(\mathrm{t})$ also comprises an identity matrix (1) of size 4 , biarticularity matrix $(\mathbf{E})$, and $\beta_{\mathrm{j}}^{\mathrm{nf}}$ and $\delta_{\mathrm{j}}^{\mathrm{nf}}$ terms to take into account that EMG amplitudes may change depending on the origins and insertions of the muscles, respectively, as well as the length and the shortening velocity of the muscle fibers. Additional constraints (Eq. (3)) were added to the procedure to ensure that the sign convention of the outputs (i.e., the contribution of the flexor and extensor muscle groups to the net joint moment) is fulfilled at each time $\mathrm{t}[8]$.

2.5.3 Dynamic Trials-Fatigued Case. A schematic representation of the methodology used in the present study to estimate the contributions of the knee flexor and extensor muscle groups to the net joint moment under fatigue condition is presented in Fig. 2.

In the fatigued condition, the formulation of the optimization problem included a supplementary equality constraint that took into account the loss of moment production capacity of the extensor muscle group. The loss of moment production capacity was estimated using the previously described linear relationship (see the isometric fatigue session section) with the normalized decrease in the EMG mean frequency between the dynamic nonfatigued and fatigued sessions as input.

The time-frequency spectrums of the EMG signals were obtained from wavelet transforms of the EMG bursts within fixedlength windows for each squat cycle. These windows of 512 samples were centered at the time instants, where the knee joint angle trajectory was the most repeatable, that is the time instants corresponding to the minimum of the knee angle standard deviation (quasi-cyclostationarity hypothesis [5]). The time-frequency spectrum estimates were then averaged over all the cycles to obtain a single estimate of the mean frequency for the selected muscle in either the nonfatigued or the fatigued condition (Fig. 3). The normalized mean frequency decrease was assessed as the dif- 


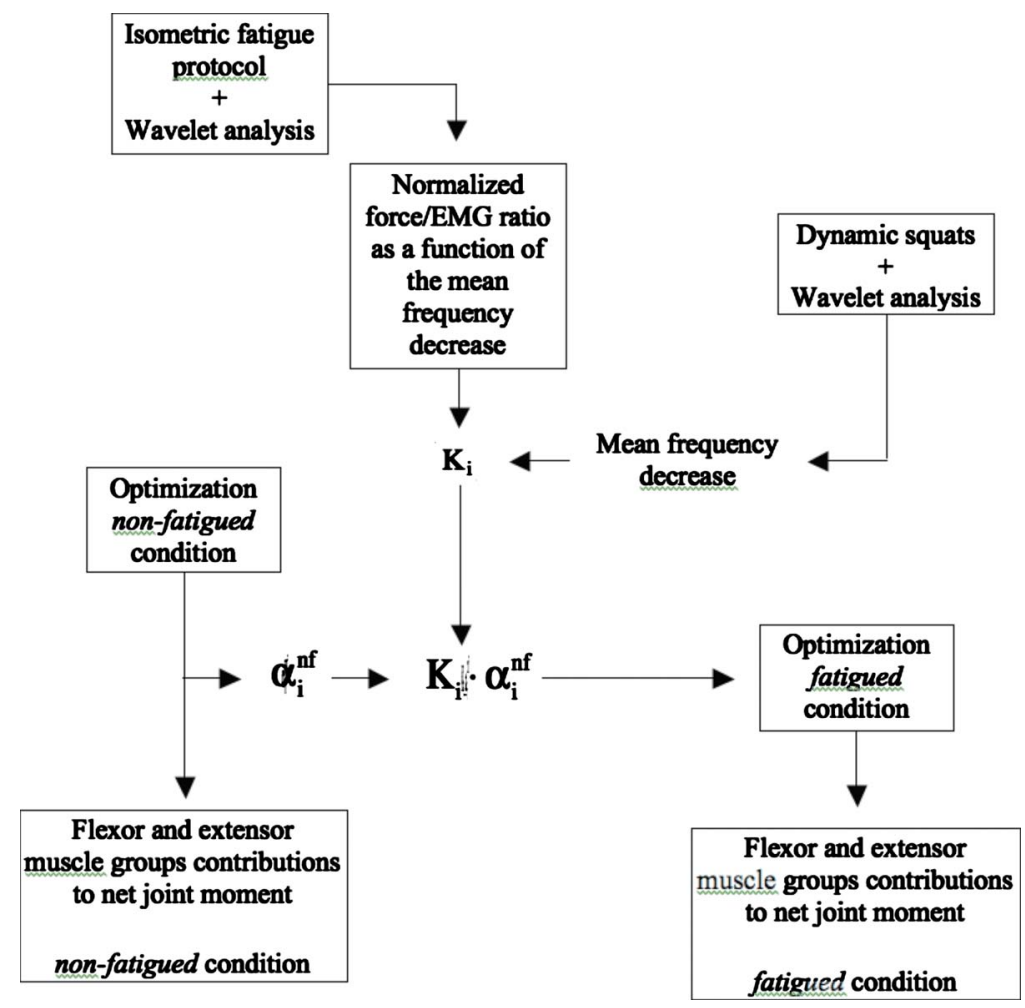

Fig. 2 Schematic representation of the methodology used in the study to assess the contributions of the flexor and extensor muscle groups to the net joint moment under fatigue condition

ference between the mean frequency estimates obtained from the nonfatigued and the fatigued conditions normalized by the value in the nonfatigued case. Using the linear regression previously created (see the isometric fatigue session section), an estimate of the moment production capacity of the fatigued muscle (the $\mathrm{K}$ coefficient) was obtained for the dynamic squat in the fatigued condition.

In the fatigued condition, the nonlinear constrained optimization problem was formulated as for the nonfatigued case (see Eqs. (1)-(3)) with a supplementary set of equality constraints (Eq. (4)). Hence, the methodology developed in this study to account for the presence of fatigue in the EMG signals was used to constrain the moment production capacity of the extensor muscle group during the resolution of the optimization problem in the fatigued case.

$$
\begin{gathered}
\alpha_{\mathrm{ga}}^{\mathrm{f}}=\alpha_{\mathrm{ga}}^{\mathrm{nf}}, \quad \alpha_{\mathrm{bf}}^{\mathrm{f}}=\alpha_{\mathrm{bf}}^{\mathrm{nf}} \\
\alpha_{\mathrm{rf}}^{\mathrm{f}}=\mathrm{K}_{\mathrm{rf}} \cdot \alpha_{\mathrm{rf}}^{\mathrm{nf}}, \quad \alpha_{\mathrm{vm}}^{\mathrm{f}}=\mathrm{K}_{\mathrm{vm}} \cdot \alpha_{\mathrm{vm}}^{\mathrm{nf}}
\end{gathered}
$$

where the $\alpha_{\mathrm{i}}^{\mathrm{f}}$ coefficient represents the isometric EMG-moment relationship in the fatigued condition and the $\mathrm{K}$ coefficients are computed from the mean frequency decrease in the EMG signals (see the dynamic trials section).

The optimization problems under the nonfatigued and fatigued conditions were solved using a sequential quadratic programming (SQP) approach [19]. All computations were done using MATLAB software (version 7; Optimization Toolbox, version 3.0.3; Spline Toolbox, version 3.2.2; Time-Frequency Toolbox).

2.6 Statistics. One-way ANOVAs (fatigue effect) were conducted on the minimum, maximum, and mean values of the ankle, knee and hip angular displacements and velocities, as well as on the model parameters. One-way ANOVAs (fatigue effect) were also carried out on the minimal, maximal, and mean values of the knee net joint moment and the contributions of the flexor and extensor muscle groups to the net joint moment. A significance level of 0.05 was used for all comparisons. Tukey post-hoc tests were used whenever necessary.

\section{Results}

3.1 Isometric Fatigue Session. The presence of muscle fatigue during the isometric session was revealed by an increase in the EMG amplitudes and a decrease in the EMG mean frequencies. Indeed, mean RMS EMG increased by $4.6 \pm 27.2 \%$ and $16.4 \pm 23.7 \%$, respectively, for the rectus femoris and the vastus medialis muscles. As mentioned in the Sec. 2, no estimate of the gastrocnemius medialis and biceps femoris mean frequencies was performed due to the low activation level of these muscles and the high variability of this variable under such conditions. Oppositely, decreases in mean frequencies of $36.0 \pm 5.9 \%$ and $23.4 \pm 12.5 \%$ for the rectus femoris and the vastus medialis muscles, respectively, were observed. These changes corresponded to decreases of $31.8 \pm 19.2 \%$ and $40.1 \pm 14.8 \%$ of the normalized force/EMG ratio for the rectus femoris and the vastus medialis muscles. During this isometric fatigue session, the knee joint angles showed little variations with maximal discrepancies below $5 \mathrm{deg}$.

An example of the original methodology developed in this study and consisting of a linear regression between the force production capacity (i.e., the normalized force/EMG ratio) and the normalized mean frequency decrease is illustrated in Fig. 4. All the slopes of the linear regressions were positive with mean values of $0.91 \pm 0.69$ and $1.28 \pm 0.52$, respectively, for the rectus femoris and the vastus medialis muscles with corresponding mean coefficients of determination $\left(r^{2}\right)$ of $0.70 \pm 0.40$ and $0.63 \pm 0.38$.

3.2 Dynamic Trials. A significant augmentation in the EMG amplitudes was seen between the nonfatigued and the fatigued dynamic conditions with an averaged increase of $29.74 \pm 15.39 \%$, 


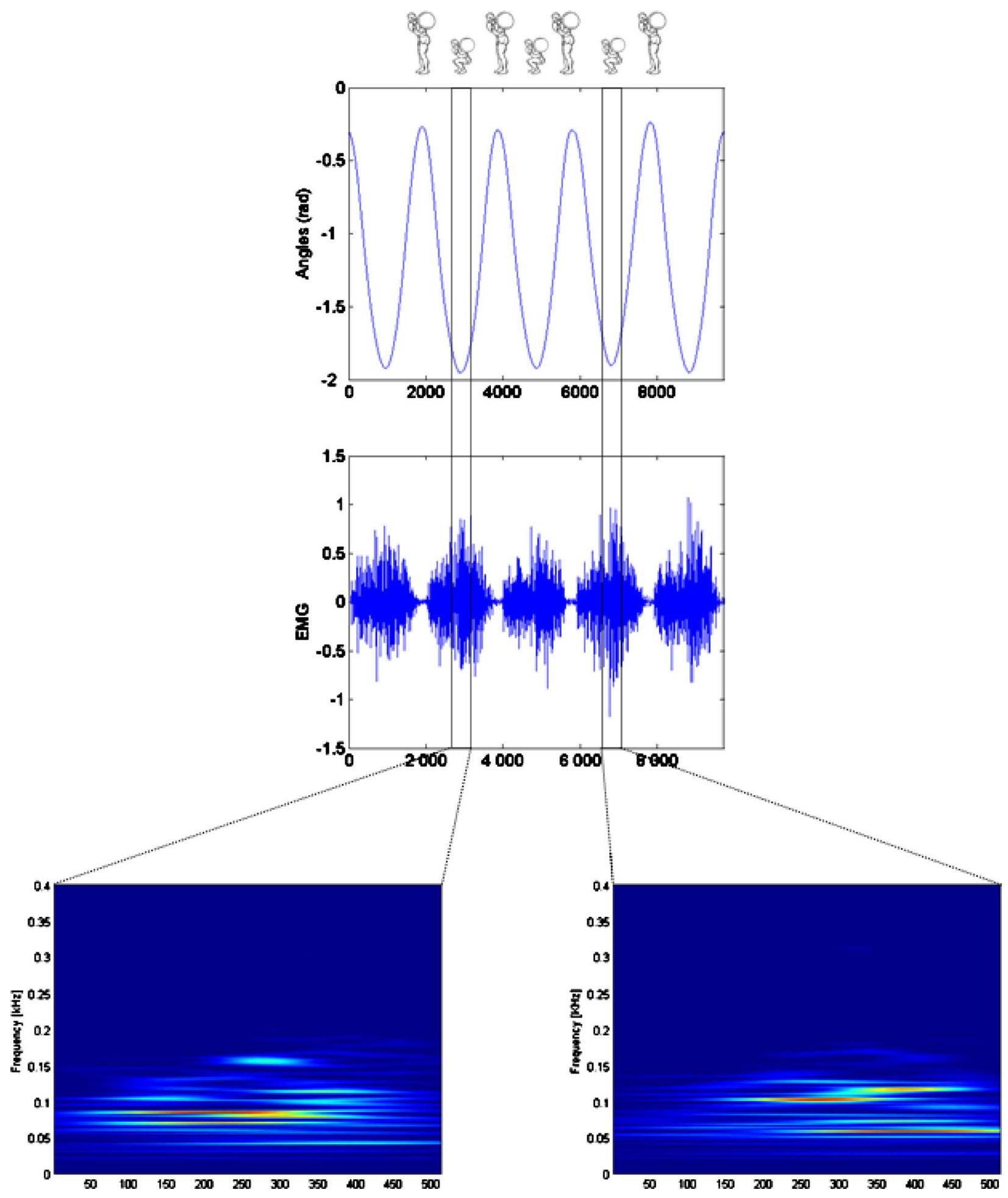

Fig. 3 Schematic representation of the methodology used to estimate the frequency content of the EMG signal. Upper graph shows the evolution through time of the knee angle. Middle graph represents the EMG signal of the rectus femoris muscle. Bottom graphs show the outputs of the wavelet analysis. The $\mathbf{5 1 2}$ points analysis windows were centered at the time instants were the knee joint angle trajectory was the most repeatable. For the bottom graphs, the time is along the horizontal axis and the frequency along the vertical one. The amplitude of the signal at any given time and frequency is represented using color scale. Brighter colors inform that the corresponding frequency bandwidth is often found in the signal frequency content.

$18.02 \pm 12.50 \%, 23.63 \pm 25.17 \%$, and $21.70 \pm 15.12 \%$ for the gastrocnemius medialis, biceps femoris, rectus femoris, and vastus medialis muscles, respectively, $\left(\mathrm{F}_{1,34}>33.02\right.$ and $\mathrm{p}<0.05$ for all muscles). Despite these modifications, kinematic data of the squat cycles were very similar between the nonfatigued and the fatigued dynamic conditions. Indeed, no statistical influence of the fatigue factor was found on the minimum, mean, and maximum values of the ankle, knee, and hip joint angles and angular velocities. More- 


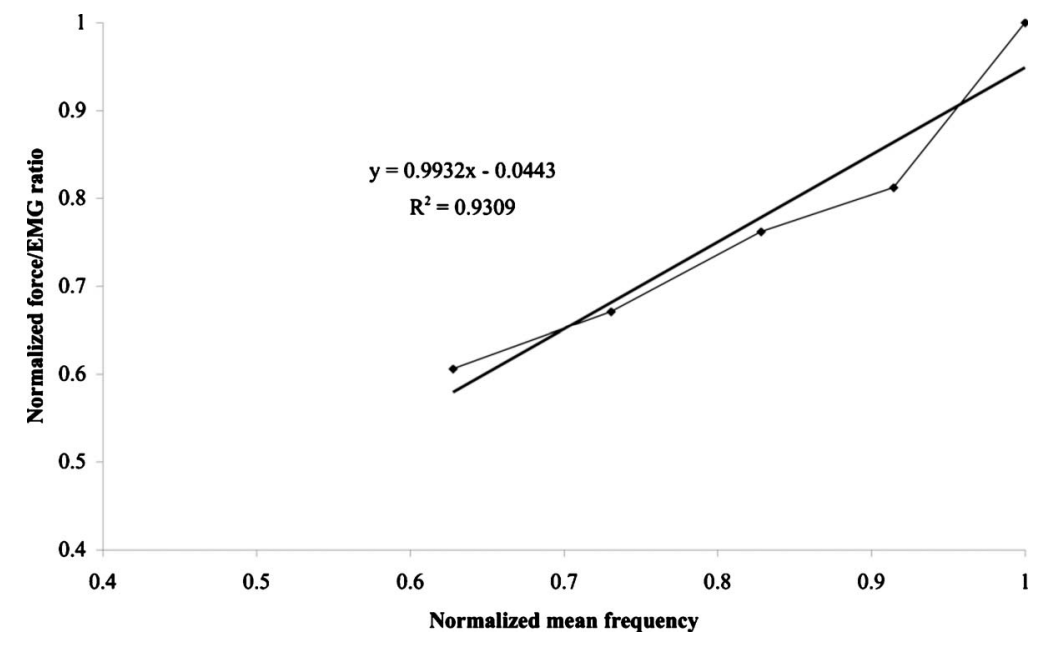

Fig. 4 Representative example for one subject of the isometric linear relationship that links the force production capacity of the rectus femoris muscle (normalized force/EMG ratio) to the normalized mean frequency decrease. Raw data as well as the corresponding linear regression are shown.

over due to the position of the bar, no motion of the upper body was allowed during the squat movement, strengthening the results that the squats were very similar in nonfatigue and fatigue conditions.

The EMG mean frequencies estimated through wavelet transforms during the dynamic squats revealed a significant decrease between the nonfatigued and the fatigued conditions with relative diminutions of $18.28 \pm 7.45 \%$ and $7.72 \pm 3.14 \%$ for the rectus femoris and the vastus medialis muscles, respectively. Once the normalized mean frequency decreases were input to the linear regressions, the moment production capacities ( $\mathrm{K}$ coefficient in Eq. (4)) were estimated to be $82.99 \pm 10.79 \%$ and $87.15 \pm 9.81 \%$ of the nonfatigued condition for the rectus femoris and vastus medialis muscles, respectively. The moment production capacity of these muscles was significantly affected by the presence of fatigue $\left(\mathrm{F}_{1,34}=33.44\right.$ and $\left.\mathrm{p}<0.05\right)$.

Despite the presence of fatigue, the EMG-driven optimization model revealed little difference in muscle group contributions to joint moments between the nonfatigued and the fatigued dynamic

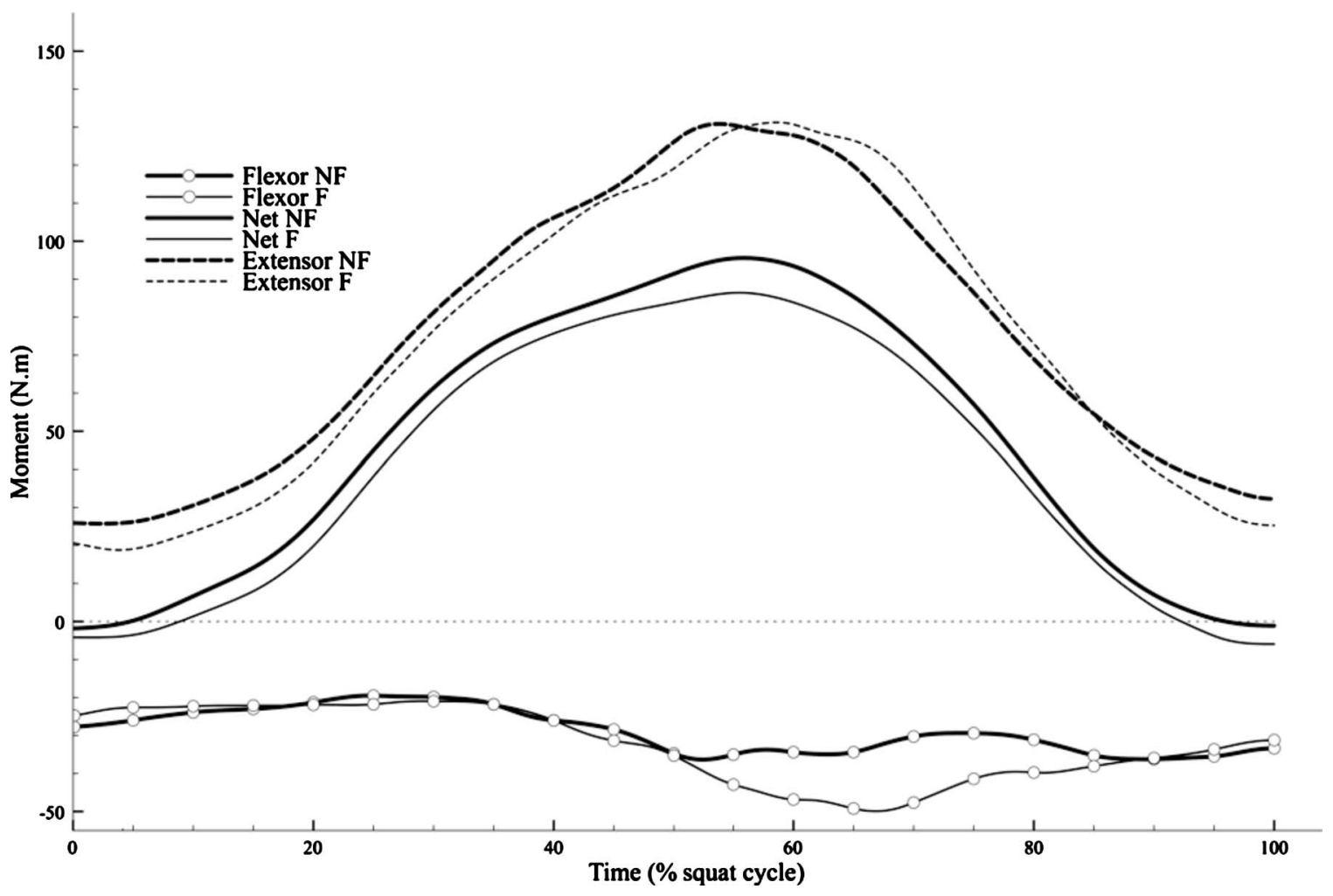

Fig. 5 Evolution through time of the mean values $(n=9)$ of the moments developed by the extensor muscle group (upper curves), the net joint moments (middle curves) and the flexor muscle group moments (lower curves). For each variable, thick lines correspond to the nonfatigued condition and thin lines to the fatigued situation. 
Table 1 Mean values ( \pm s.d.) of the optimized parameters. $\alpha_{\mathrm{i}}$, $\beta_{\mathrm{i}}$, and $\delta_{\mathrm{i}}$ represent, respectively, the isometric EMG-moment coefficient and the terms accounting for the force-length and force-velocity relationships. ${ }^{*}$ indicates a significant influence of the fatigue factor $(p<0.05)$.

\begin{tabular}{lccc}
\hline \hline & $\mathrm{NF}$ & $\mathrm{F}$ \\
\hline$\alpha_{\mathrm{ga}}$ & $-80.67 \pm 50.51$ & $-80.67 \pm 50.51$ & \\
$\alpha_{\mathrm{bf}}$ & $-54.81 \pm 25.89$ & $-54.81 \pm 25.89$ & $*$ \\
$\alpha_{\mathrm{rf}}$ & $154.29 \pm 55.15$ & $123.28 \pm 45.94$ & $*$ \\
$\alpha_{\mathrm{vm}}$ & $150.76 \pm 54.32$ & $130.95 \pm 51.08$ & $*$ \\
$\beta_{\mathrm{A}}$ & $0.80 \pm 0.35$ & $0.95 \pm 0.51$ & $*$ \\
$\beta_{\mathrm{K}}$ & $0.19 \pm 0.09$ & $0.09 \pm 0.07$ & \\
$\beta_{\mathrm{H}}$ & $0.35 \pm 0.13$ & $0.38 \pm 0.12$ & \\
$\delta_{\mathrm{A}}$ & $0.14 \pm 0.06$ & $0.16 \pm 0.09$ & \\
$\delta_{\mathrm{K}}$ & $0.04 \pm 0.01$ & $0.03 \pm 0.02$ & \\
$\delta_{\mathrm{H}}$ & $0.06 \pm 0.03$ & $0.07 \pm 0.05$ & \\
\hline \hline
\end{tabular}

conditions (Fig. 5). Indeed, the minimum, maximum, and mean values of the knee net joint moments showed no difference due to the fatigue factor. No statistical influence of the fatigue factor was seen on the contributions of the flexor and extensor muscle groups to the net joint moment estimated with either the original version of the model (nonfatigued condition) or the version that included the equality constraint ( $\mathrm{K}$ coefficient) on the moment production capacity (fatigued condition).

The comparison of the parameters issued from the optimization processes (Eq. (1)-(4)) revealed no influence of the fatigue factor (Table 1), except on the $\beta_{\mathrm{K}}$ term (i.e., the one that accounts for the changes in the EMG amplitudes due to the force-length relationship at the knee joint). The influence of the fatigue factor was also nonsignificant for the $\mathrm{w}_{\mathrm{i}}(\mathrm{t})$ terms. Indeed, the evolutions through time of the $\mathrm{w}_{\mathrm{i}}(\mathrm{t})$ coefficients were similar between the nonfatigued and the fatigued dynamic conditions (Fig. 6).

\section{Discussion}

The aim of the present study was to develop an EMG-driven biomechanical model to obtain the contributions of flexor and extensor muscle groups to the net joint moment during a nonisokinetic functional movement (squat exercise) performed in nonfatigued and in fatigued conditions. An EMG-based methodology that aims at balancing the decreased muscle moment production capacity following fatigue was developed. The outputs of the new

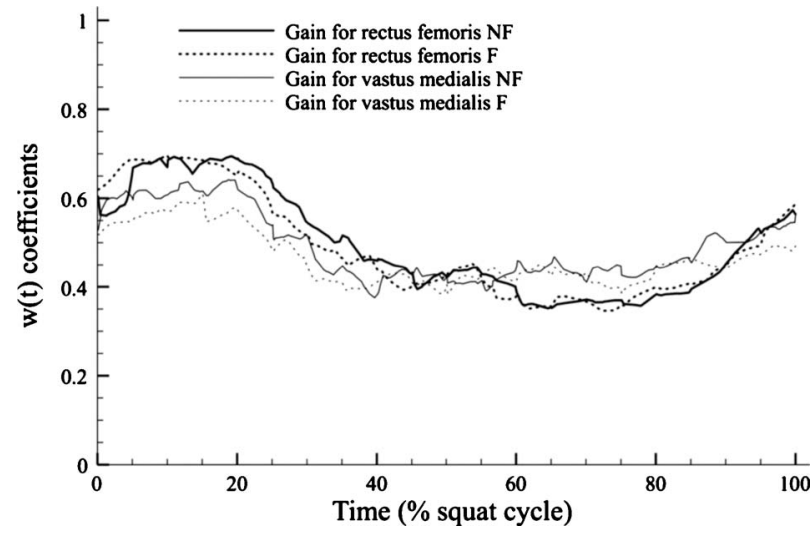

Fig. 6 Evolution through time of the individual muscle gains of the rectus femoris (thick lines) and vastus medialis (thin lines) muscles in the nonfatigued (continuous lines) and fatigued (dashed lines) situations. Note the similar patterns of the $w_{i}(t)$ coefficients, which imply that the optimization procedure did not take advantage of this variable to compensate for the additional equality constraint. methodology indicated that the decreases in mean frequency during the fatigued dynamic squats corresponded to moment production capacities of $83 \%$ and $87 \%$ of the nonfatigued values for the rectus femoris and the vastus medialis muscles, respectively. These values are consistent with the fact that the maximal force depletion was $33 \%$ at the end of the isometric fatigue session. It was demonstrated that the model was able to characterize fatigue with a single coefficient for each muscle $\mathrm{K}_{\mathrm{i}}$, which accounted for fatigue-induced frequency shifts obtained using wavelet analysis.

Our working hypothesis stated that with identical kinematics and dynamics data and different EMG inputs, the proposed methodology could estimate the decreased muscle moment production capacity due to fatigue and take this decrease into account in the EMG-to-moment procedure. While the outputs of the optimization procedure were similar, the optimization parameters (Eqs. (2)-(4)) could have been modified during the resolution by the SQP algorithm. The consistency of the present methodology was reinforced by the lack of significant differences between the optimized parameters of the model (compare Table 1), except for the term accounting for the force-length relationship at the knee joint (i.e., $\beta_{\mathrm{K}}$ ). However, the difference on the $\beta_{\mathrm{K}}$ coefficient is not surprising since a dependence of the force-length relationship to muscle fatigue as been reported by [20]. The stability of the optimized parameters revealed that the optimization procedure did not take advantage of the other variables to compensate for the additional equality constraint. This point is supported by the similar patterns of the $\mathrm{w}_{\mathrm{i}}(\mathrm{t})$ coefficients (compare Fig. 6).

Our results showed no influence of the fatigue factor on the flexor and extensor contributions to the net joint moment, though a general increase in the EMG activities was seen between the nonfatigued and the fatigued dynamic conditions. The procedure developed in this study and included in an EMG-driven model aimed at taking into account the changes in the EMG amplitudes due to muscle fatigue (i.e., the changes in the activation level) to estimate the contributions of the knee flexor and extensor muscle group to the net joint moment. Expectedly with no influence of the fatigue factor on the kinematic data, the net knee joint moments were very similar between the nonfatigued and the fatigued sessions. As the net joint torques required to produce the task in both conditions were similar, the central nervous system increased the activation of the fatigued muscle group to reach the necessary extension moment. An increased activation of the agonist group could lead to increased antagonist activation because of a common drive to the agonist-antagonist pairs [21]. In the present study, higher EMG amplitudes were also seen for the antagonist muscle group reinforcing the "common drive" hypothesis. However, as previously stated in Ref. [22] during squats with low loads, the activation levels of antagonist muscles were low (mean values below 10\% MVF) and a relative 25\% increase in the EMG amplitudes would have little effects on the muscle group moment. The outputs of the EMG-to-moment optimization process showed no difference in the antagonist contribution to the net joint moment between the nonfatigued and the fatigued conditions. Hence, while the EMG of the extensor muscle group is logically increased, the contribution of the extensor muscle group is kept constant through the different conditions. These results satisfied our hypothesis in that the developed procedure was able to take into account the changes in the activation level of the fatigued muscles for their use in an EMG-driven model in the fatigued condition.

The procedure described in this study mainly relied on the estimation of the EMG mean frequencies both during isometric and dynamic contractions. Several factors may influence the estimation of the frequency content of the EMG signals (i.e., nonstationary feature of the EMG signal, muscle activation levels, muscle fiber length, muscle fiber velocity). Although the use of short time fast fourier transform (FFT) may have provided the same results [23], it is likely that the use of wavelet transforms to estimate the EMG frequency content in fatigued isometric and dynamic condi- 
tions will enhance the accuracy of the spectral estimates $[5,6]$. The other possible artifacts were also carefully taken into account throughout this study. First, the isometric session was performed at $60 \% \mathrm{MVC}$ and the EMG analysis windows during the dynamic sessions were located at time instants of high activation (compare Fig. 3), thus removing the activation level as a possible artifact in the estimation of the mean frequencies [24]. Second, the frequency estimates had also shown a strong dependence on muscle fiber length [25]. In our experiment, the variations in knee joint angle (and consequently in muscle length) during the isometric fatigue session were less than 5 deg for all the subjects. During the dynamic sessions, the spectral estimates were carried out within analysis windows located at time instants, where the knee joint angle trajectories were the most repeatable. No influence of the fatigue factor was seen on the kinematics data and the knee angles within the analysis windows were similar between the nonfatigued and the fatigued sessions. Thus, EMG frequency content was estimated at the same muscle length for the nonfatigued and the fatigued dynamic conditions. Third, the time instants, where the knee joint angles were the most repeatable also corresponded to a knee joint velocity close to zero. The EMG mean frequencies in dynamic conditions were thus estimated during a "quasiisometric" contraction, avoiding the artifacts due to differences in the muscle fiber shortening velocity.

The isometric fatigue session was used to create a linear regression between the moment production capacity of the muscles (i.e., the normalized force/EMG ratio) and the decrease in mean frequency. Indeed, the force production capacity has been shown to decrease linearly with fatigue during submaximal exercises [26] and previous work had represented the mean frequency decrease through time using a linear model and reported a good fit with the experimental data [27]. However, some subjects showed low coefficients of determination for the linear regression (with a $\mathrm{r}^{2}$ value of 0.12 for the worst case). In future studies several possibilities are offered to improve these values. First, using different regressions than the linear one (i.e., polynomial, exponential, and logarithmic) may improve the values of the coefficient of determination. Indeed under fatigue condition, previous studies reported that some of physiologic parameters evolved with a nonlinear trend [28]. Second, as the regression was created from raw force/EMG ratios and mean frequency data, this issue may be resolved by modeling previously, and separately, the evolutions through time of the normalized force/EMG ratio and of the mean frequency decrease. Afterward, the previous modeled data could be put all together to create the final regression between the moment production capacity and the decrease in mean frequency. While the present experiment was conducted using an isometric fatigue session, the linear regression between the muscular force production capacity and the mean frequency could be obtained from dynamic fatiguing exercises. Indeed, during a long lasting dynamic exercise, consistent and quasi-linear mean frequency decreases were reported when studying the same joint angle range over the entire range of motion during the fatiguing exercise [25]. This point enhances the applicability of the methodology to different types of muscle fatigue as isometric exercises or repetitive exertion bouts both lead to a decrease in EMG mean frequency and an increase in EMG amplitude for a constant force output.

While some limitations may appear as the choice of the representative muscles or the fact that the level of fatigue of the knee flexors could not be tested, we believe that the methodology developed in this study has several advantages. First, to our knowledge, this work is among the first in attempting to estimate the force production capacity of fatigued muscles during functional dynamic nonisokinetic movements. This feature extends the use of the EMG-driven models to functional activities performed under muscle fatigue. As previous work reported changes in muscle activation patterns during cutting movements following muscle fatigue [29], such a procedure can help in studying the stress experienced by the ligaments in situations usually encountered during sport exercises. Second, while the described procedure can be applied generally to a wide range of activities and EMG-driven models, the subject-specific trend of the development of muscle fatigue is fully taken into account. Indeed, the linear regression built during an isometric or dynamic fatigue session is tuned specifically to the properties of each muscle.

In conclusion, this study presented an original procedure based on the decrease in the frequency content of the EMG data to assess the force production capacity of a muscle group following a fatiguing exercise. Based on this procedure, an equality constraint was added to an EMG-driven biomechanical model to estimate the contributions of the flexor and extensor muscle group to the net joint moment during dynamic exercise under fatigue condition. The results presented here are promising, as they constitute the first attempt to estimate the force production capacity of a fatigued muscle group during a dynamic, nonisokinetic exercise. The procedure previously described may find applications for the study of several functional activities performed under muscle fatigue such as rehabilitation exercises, walking, jogging, etc. Further studies are necessary to extend the use of the proposed method to other biomechanical models. In particular, more detailed models that aimed at estimating muscle forces [9] could benefit in the use of the procedure described in this study.

\section{References}

[1] Basmajian, J. V., and De Luca, C. J., 1985, Muscle Alive, 5th ed., Williams and Wilkins, Baltimore.

[2] Gandevia, S. C., 2001, "Spinal and Supraspinal Factors in Human Muscle Fatigue," Physiol. Rev., 81, pp. 1725-1789.

[3] Cheng, A. J., and Rice, C. L., 2005, "Fatigue and Recovery of Power and Isometric Torque Following Isotonic Knee Extensions,” J. Appl. Physiol., 99, pp. 1446-1452.

[4] Westerblad, H., and Allen, D. G., 2002, "Recent Advances in the Understanding of Skeletal Muscle Fatigue," Curr. Opin. Rheumatol., 14, pp. 648-652.

[5] Bonato, P., Roy, S. H., Knaflitz, M., and De Luca, C. J., 2001, "TimeFrequency Parameters of the Surface Myoelectric Signal for Assessing Muscle Fatigue During Cyclic Dynamic Contractions," IEEE Trans. Biomed. Eng., 48, pp. 745-753

[6] Karlsson, J. S., Ostlund, N., Larsson, B., and Gerdle, B., 2003, “An Estimation of the Influence of Force Decrease on the Mean Power Spectral Frequency Shift of the EMG During Repetitive Maximum Dynamic Knee Extensions," J. Electromyogr. Kinesiol., 13, pp. 461-468.

[7] Buchanan, T. S., Almdale, D. P., Lewis, J. L., and Rymer, W. Z., 1986, "Characteristics of Synergic Relations During Isometric Contractions of Human Elbow Muscles," J. Neurophysiol., 56, pp. 1225-1241.

[8] Amarantini, D., and Martin, L., 2004, "A Method to Combine Numerical Optimization and EMG Data for the Estimation of Joint Moments Under Dynamic Conditions," J. Biomech., 37, pp. 1393-1404.

[9] Buchanan, T. S., Lloyd, D. G., Manal, K., and Besier, T. F., 2005, "Estimation of Muscle Forces and Joint Moments Using a Forward-Inverse Dynamics Model," Med. Sci. Sports Exercise, 37, pp. 1911-1916.

[10] Cholewicki, J., McGill, S. M., and Norman, R. W., 1995, "Comparison of Muscle Forces and Joint Load From an Optimization and EMG Assisted Lumbar Spine Model: Towards Development of a Hybrid Approach,” J. Biomech., 28, pp. 321-331.

[11] Sparto, P. J., and Parnianpour, M., 1998, "Estimation of Trunk Muscle Forces and Spinal Loads During Fatiguing Repetitive Trunk Exertions," Spine, 23, pp. $2563-2573$.

[12] Olney, S. J., and Winter, D. A., 1985, "Predictions of Knee and Ankle Moments of Force in Walking From EMG and Kinematic Data," J. Biomech., 18, pp. 9-20.

[13] Hermens, H. J., Freriks, B., Disselhorst-Klug, C., and Rau, G., 2000, "Development of Recommendations for SEMG Sensors and Sensor Placement Procedures," J. Electromyogr. Kinesiol., 10, pp. 361-374.

[14] Winter, D. A., 1990, Biomechanics and Motor Control of Human Movements, Wiley-Interscience, Toronto, Ontario.

[15] Zatsiorsky, V., and Seluyanov, V., 1983, "The Mass and Inertial Characteristics of Main Segments of the Human Body," Biomechanics VIII-B, Human Kinetics, Champaign, pp 1152-1159.

[16] Karlsson, J. S., and Gerdle, B., 2001, "Mean Frequency and Signal Amplitude of the Surface EMG of the Quadriceps Muscles Increase With Increasing Torque-A Study Using the Continuous Wavelet Transform,” J. Electromyogr. Kinesiol., 11, pp. 131-140.

[17] Auger, F., Flandrin, P., Goncalves, P., and Lemoine, O., 1996, "TimeFrequency Toolbox-for Use With Matlab," Reference Guide, Centre National de la Recherche Scientifique, France.

[18] Centomo, H., Amarantini, D., Martin, L., and Prince, F., 2007, "Differences in the Coordination of Agonist and Antagonist Muscle Groups in Below-Knee Amputee and Able-Bodied Children During Dynamic Exercise," J. Electromyogr. Kinesiol., 18, pp. 487-494. 
[19] Boggs, P. T., and Tolle, J. W., 1995, "Sequential Quadratic Programming," Acta Numerica, 4, pp. 1-52.

[20] Butterfield, T. A., and Herzog, W., 2005, "Is the Force-Length Relationship a Useful Indicator of Contractile Element Damage Following Eccentric Exercise?," J. Biomech., 38, pp. 1932-1937.

[21] De Luca, C. J., and Mambrito, B., 1987, "Voluntary Control of Motor Units in Human Antagonist Muscles: Coactivation and Reciprocal Activation," J. Neurophysiol., 58, pp. 525-542.

[22] Isear, J. A., Erickson, J. C., and Worrell, T. W., 1997, "EMG Analysis of Lower Extremity Muscle Recruitment Patterns During an Unloaded Squat," Med. Sci. Sports Exercise, 29, pp. 532-539.

[23] Maclsaac, D. T., Parker, P. A., Scott, R. N., Englehart, K. B., and Duffley, C., 2001, "Influence of Dynamic Factors on Myoelectric Parameters," IEEE Eng. Med. Biol. Mag., 20, pp. 82-89.

[24] Linnamo, V., Strojnik, V., and Komi, P. V., 2002, "EMG Power Spectrum and Features of the Superimposed M-Wave During Voluntary Eccentric and Concentric Actions at Different Activation Levels,” Eur. J. Appl. Physiol., 86, pp. 534-540.
[25] Potvin, J. R., 1997, "Effects of Muscle Kinematics on Surface EMG Amplitude and Frequency During Fatiguing Dynamic Contractions," J. Appl. Physiol., 82, pp. 144-151.

[26] Stephens, J. A., and Taylor, A., 1972, "Fatigue of Maintained Voluntary Muscle Contraction in Man," J. Physiol. (London), 220, pp. 1-18.

[27] Coorevits, P. L. M., Danneels, L. A., Ramon, H., Van Audekercke, R., Cambier, D. C., and Vanderstraeten, G. G., 2005, "Statistical Modeling of FatigueRelated Electromyographic Median Frequency Characteristics of Back and Hip Muscles During a Standardized Isometric Back Extension Test," J. Electromyogr. Kinesiol., 15, pp. 444-451.

[28] Binder-Macleod, S. A., Lee, S. C. K., Fritz, A. D., and Kucharski, L. J., 1998 "New Look at Force-Frequency Relationship of Human Skeletal Muscle: Effects of Fatigue," J. Neurophysiol., 79, pp. 1858-1868.

[29] Nyland, J. A., Caborn, D. N., Shapiro, R., and Johnson, D. L., 1997, "Fatigue After Eccentric Quadriceps Femoris Work Produces Earlier Gastrocnemius and Delayed Quadriceps Femoris Activation During Crossover Cutting Among Normal Athletic Women," Knee Surg. Sports Traumatol. Arthrosc, 5, pp. 162 167. 